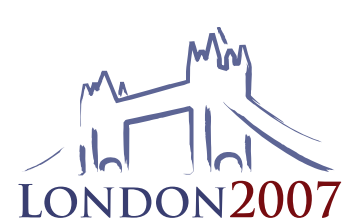

\title{
P107
}

\section{Classification of Surface Geoelectric Arrays}

\author{
S. Szalai* (Geodetic \& Geophysical Research Institute of HAS) \& L. Szarka \\ (Geodetic \& Geophysical Research Institute of HAS)
}

\section{SUMMARY}

We have found in the geophysical literature more than ninety different surface geoelectric arrays, fulfilling an updated definition (specifying the current feeding, the potential difference measurement and the geometry of the electrodes). Several composite configurations, with widely varying geometry, have also been collected. We have presented the geoelectric arrays in a systematic way and with a unified notation. The classification is based on three divalent parameters: "superposition" of measurements, "focusing" of currents and "colinearity" of the array, creating 8 groups of geoelectric arrays. For the simplest group (the group of nonfocused, nonsuperposed, colinear arrays) we cover all theoretically possible arrays. For the other groups - due to the infinite variety - we collected only the already existing arrays, but it is easy to create further example arrays. The proposed classification may facilitate a systematic comparison of properties of different arrays and inspire testing new arrays, to find optimal configurations for actual field problems. Finally, the classification certainly helps to avoid rediscovering already published arrays. 


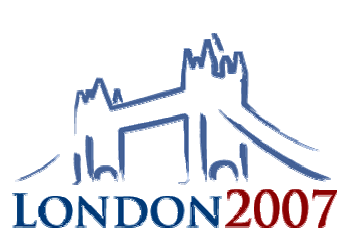

\section{INTRODUCTION}

The long history and rich variety of geoelectric resistivity measurements was summarised by Van Nostrand and Cook (1966), and the first (and so far the only) summary of geoelectric electrode arrays was published by Whiteley (1973). Many arrays from the Soviet Union are nearly completely missing from Whiteley's work. Unfortunately neither Whiteley's paper, nor these Eastern publications are easily accessible. Moreover, since the work of Whiteley (1973) the number of electrode arrays has increased significantly. Therefore it seems beneficial to provide a new classification of geoelectric electrode arrays.

With the advent of multielectrode arrays, the Wenner and dipole-dipole arrays have become the most popular arrays, and all other arrays are less frequently used. While there are important practical advantages of the Wenner and dipole-dipole arrays (e.g., multilectrode compatibility, well established theory, available inversion softwares, etc.), each specific array geometry may possess advantages worth exploiting. So it is not at all reasonable to ignore the lesser known arrays. Although there is a recent comparison study about 2D resistivity imaging of ten different electrode arrays (Dahlin and Zhou 2004), certain problems cannot be (effectively) solved by means of routine multielectrode arrays. For example, square arrays are superior over multielectrode arrays in anisotropy studies (Tsokas et al. 1997), and fissure directions can be more effectively detected by means of the Schumberger null array than by conventional arrays (Szalai et al. 2002).

In this paper we propose a new, systematic classification of geoelectric arrays, with a unified notation, and we present some examples.

\section{PRINCIPLE OF THE CLASSIFICATION}

In our classification both the current and potential electrodes are point electrodes, and all of them are supposed to be on the surface. For these surface geoelectric arrays we apply a uniform notation, and, in order to be able to compare the geometrical characteristics (e.g., the penetration depths), we defined the characteristic length of each array.

The classification of arrays is based on three divalent parameters; closely related to the components of the definition of the term "geoelectric array":

a) superposition: if the number of potential difference measurements is more than one, the array is said to be "superposed", otherwise the array is "nonsuperposed";

b) focusing: if more than one current circuit is applied, the array is said to be "focused"; otherwise the array is "nonfocused".

c) colinearity: if the alignment of the electrodes is linear, the array is said to be "colinear"; otherwise the array is "noncolinear".

In this way there are altogether $2^{3}=8$ groups, where the simpler alternative is denoted with " 1 " and the more complicated one is denoted with " $m$ ". The eight groups, shown in Fig. 1, are as follows:

I. Nonsuperposed, nonfocused, colinear arrays ("1-1-1") arrays (the "simplest" arrays)

II. Nonsuperposed, nonfocused, noncolinear ("1-1-m") arrays (the "simple noncolinear" arrays)

III. Nonsuperposed, focused, colinear ("1-m-1") arrays (the "simple focused" arrays) arrays)

IV. Superposed, nonfocused, colinear ("m-1-1") arrays (the "simple superposed"

V. Nonsuperposed, focused, noncolinear (" $1-\mathrm{m}-\mathrm{m}$ ") arrays

VI. Superposed, nonfocused, noncolinear ("m-1-m") arrays

VII. Superposed, focused, colinear (" $\mathrm{m}-\mathrm{m}-1$ ") arrays

VIII. Superposed, focused, noncolinear (“m-m-m”) arrays. 

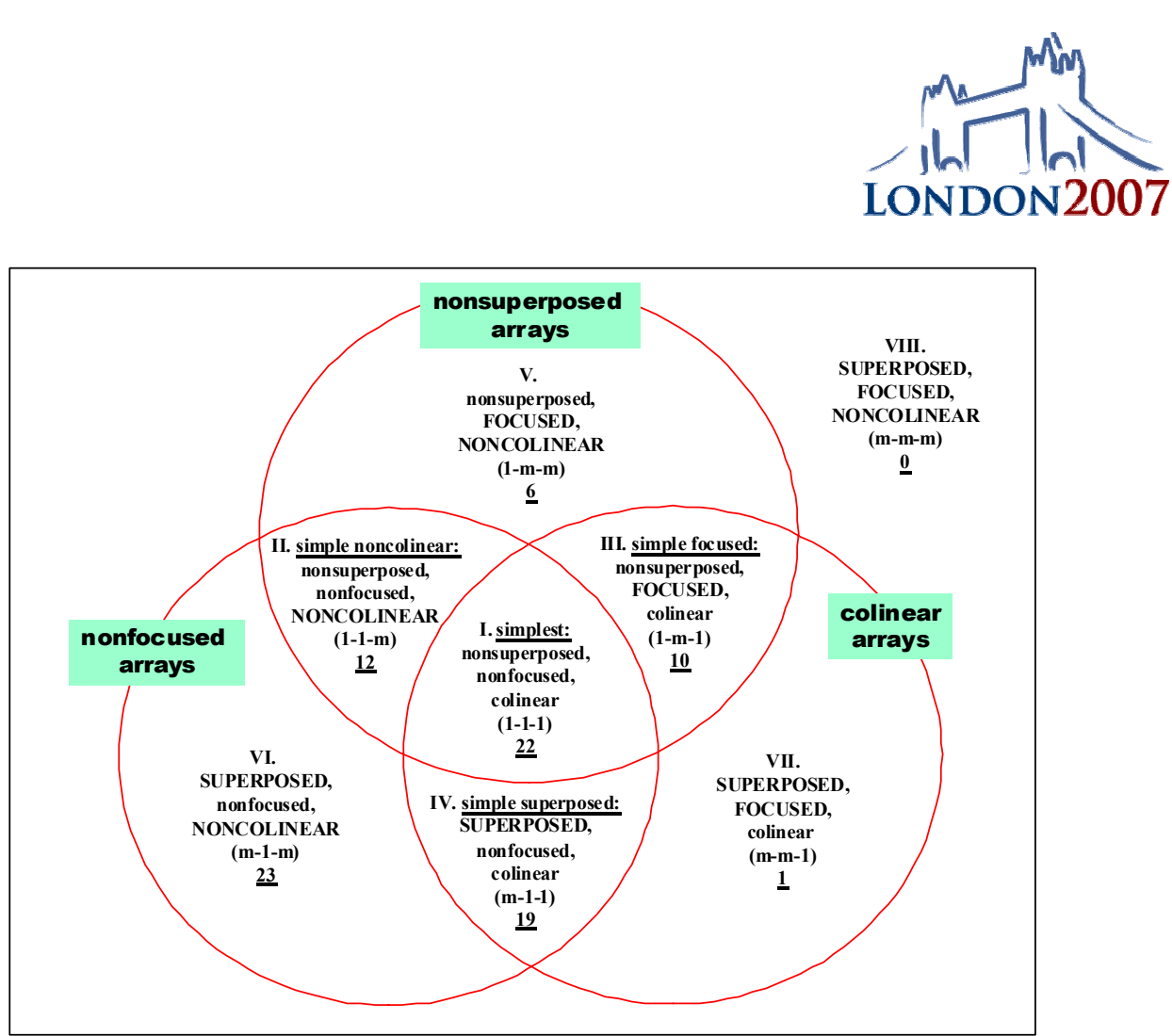

Figure 1 Classification of surface electrode arrays. Divalent (1 or $m$, where $m$ means "more") parameters as (a) superposition (nonsuperposed or superposed, that is 1 or $\mathrm{m}$ ), (b) focusing (nonfocused or focused, that is 1 or $m$ ), and (c) colinearity (colinear and noncolinear, that is 1 or $m$ ) define $2^{3}=8$ array groups as follows: 1-1-1 (group I), 1-1-m (group II), 1-m-1 (group III), 1-m-m (group IV), m-1-1 (group V), m-1-m (group VI), m-m-1 (group VII), m-m-m (group VIII).

Here we provide some selected examples: group I (in Figure 2), group V (in Figure 3), and a hypothetical array from group VIII (in Figure 4). A full list of arrays is given by Szalai and Szarka (2007).

In these figures everywhere a unified notation is used. The full circles are potential (P) electrodes, while the full/empty stars indicate source/sink (+I/-I) current (C) electrodes. (The source and sink electrodes are of course interchangeable within the same circuit, but they are not interchangeable in the case of multiple current circuits.) In a given array, the size of stars refers to the current intensity. The current electrodes at infinite distances carry unity current. The current intensity is also unity in case of all arrays, where there is only one (+I / -I) current electrode pair. The characteristic array length is defined as the longest finite distance between the electrodes. In this way the geometric characteristics, e.g., the penetration depth of different arrays becomes comparable. In the figures the characteristic array length is shown as a unit (0-1) distance. In the figures only one name is indicated for each of the arrays. All other alternative names, we have found, are summarized (together with the corresponding references) in Table 1. In Figs 2-8, if there are alternative names, the name shown in the figures (selected and suggested by the authors" of this paper for general use) is denoted by asterisk. Eventual coincidences in names are due to mutual ignorance of the authors about each other. For example, the term "dipole-dipole" has been used to describe both the "Wenner- $\beta$ " and the "dipole-axial" arrays, as well as Alpin's "dipole" array of the new classification.

The names of "null arrays" (the so called "compensation electrode arrays" by Whiteley 1973) are written in cursive. For the "null" arrays, the measured potential difference is zero over a homogeneous half-space. 


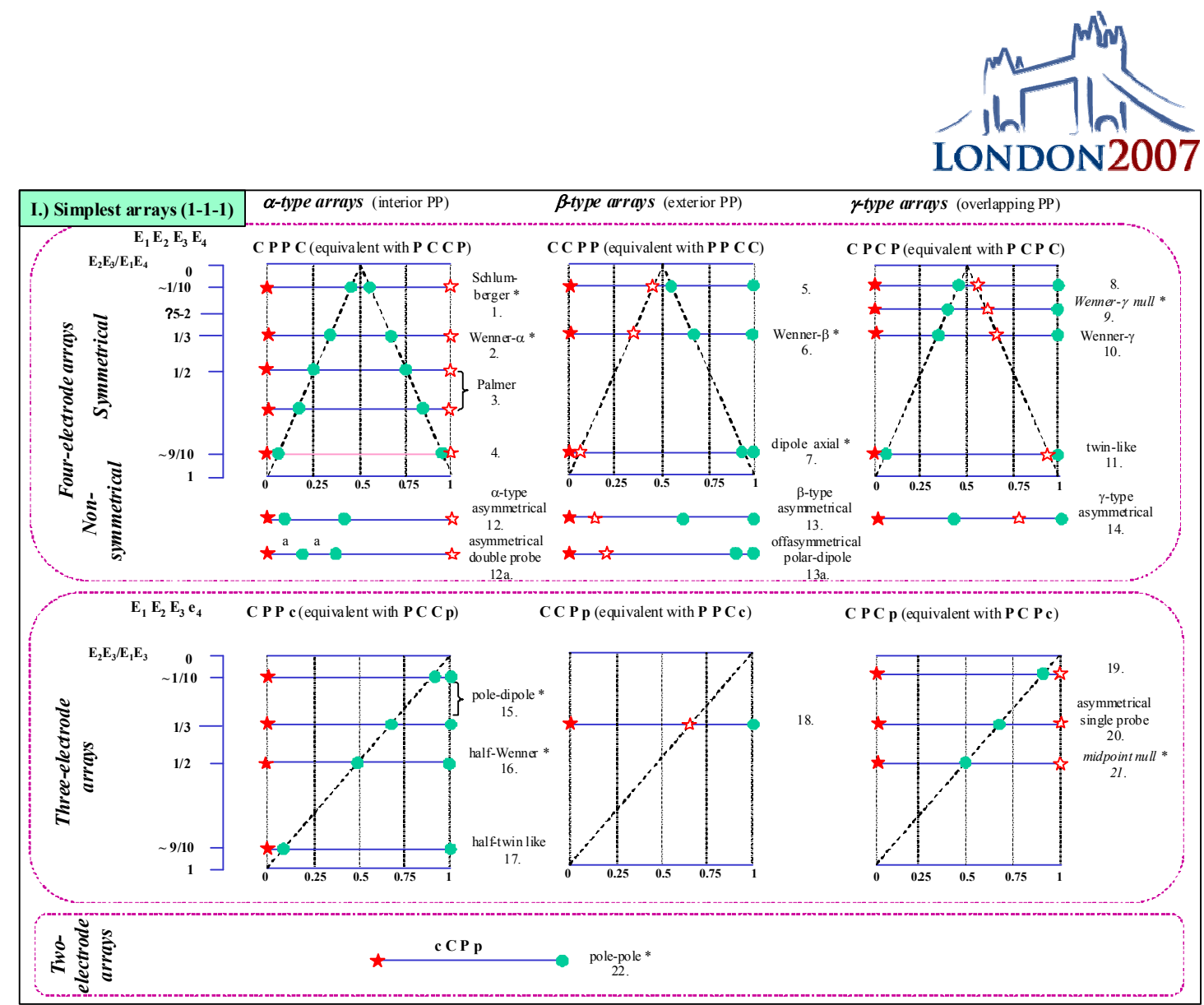

Figure 2 The "simplest" (nonsuperposed, nonfocused, colinear) geoelectric arrays (group I: 1-1-1). The electrodes in general are denoted as $E_{1}, E_{2}, E_{3}, E_{4}$. C: current (source or sink electrode). Source/sink electrodes are full/empty stars. P: potential electrode (full circles). Small letters as e, $p$, c indicate electrodes at infinity. Stars indicate alternative names (see them in Table 1); null arrays are typed in cursive.

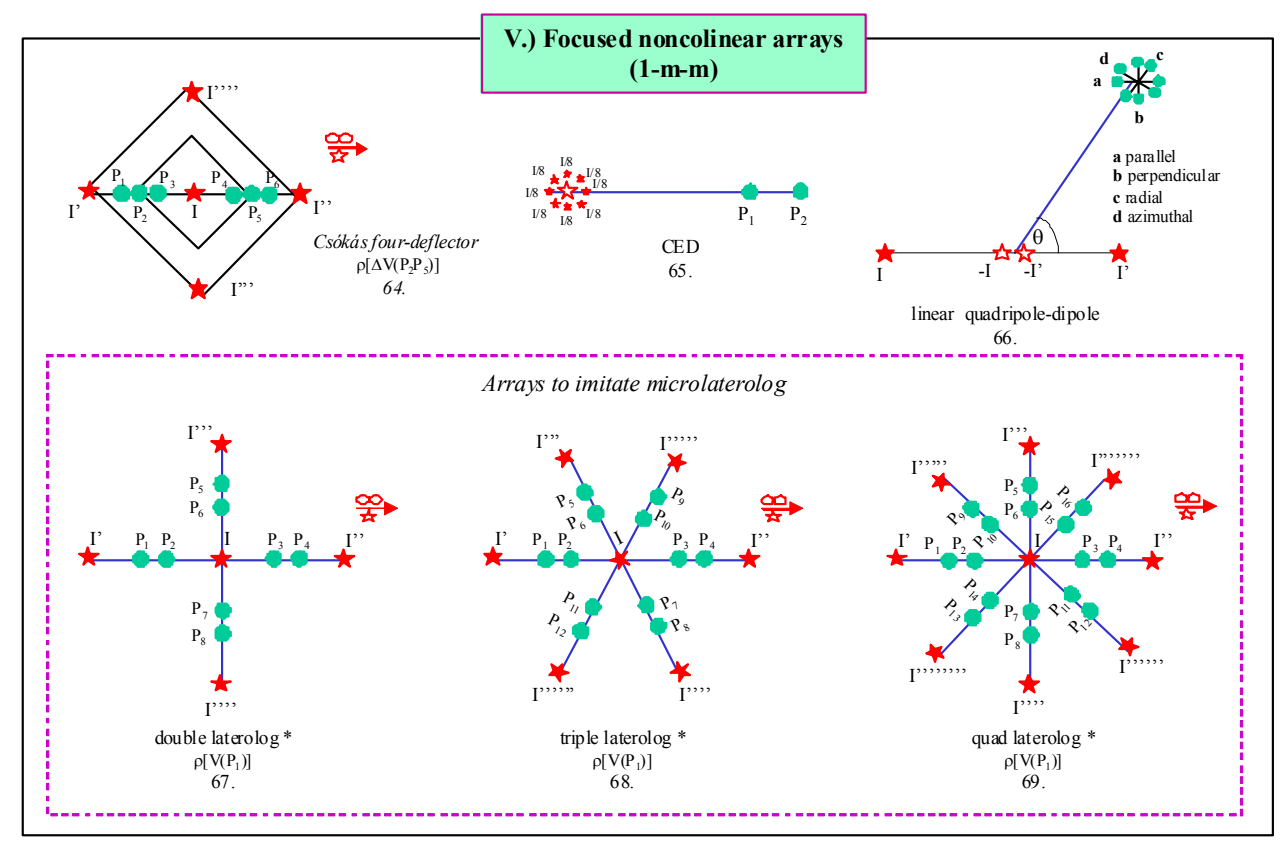

Figure 3 Nonsuperposed, focused, noncolinear arrays (group V: 1-m-m). Notations are the same as in Fig. 2. 

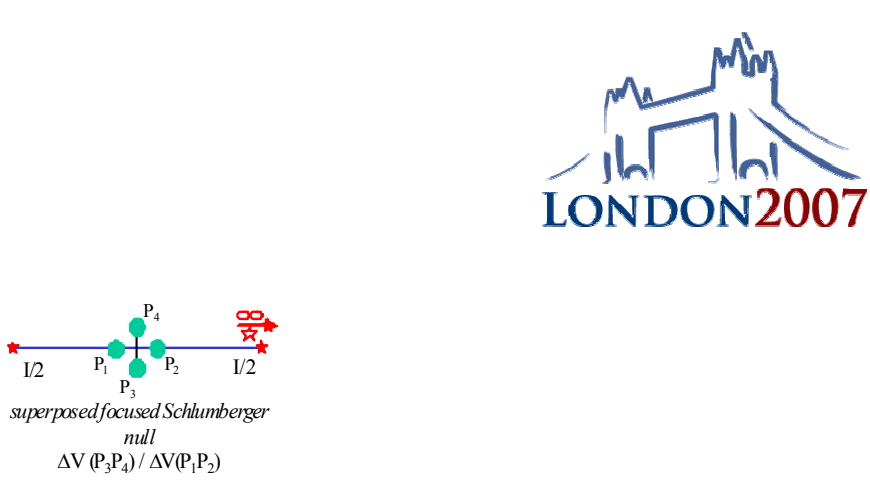

Figure 4 A hypothetical "superposed focused Schlumberger null" array in group VIII.

\section{SUMMARY}

We present all geoelectric arrays we have found in the geophysical literature, in a systematic way through a unified notation. The "composite arrays", i.e. arrays falling out of the definition of geoelectric arrays, have also been collected.

In this paper we present the first classification of more than ninety arrays, based of three divalent parameters: (1) superposition of measurements, (2) focusing of currents, (3) linearity of geometrical configuration. Having altogether 8 array groups, a very natural classification was obtained. In this way, the apparently ad hoc set of various arrays becomes a systematic geoelectric arsenal, where even the evolution of geoelectric arrays is evident.

The very first geoelectric arrays were the simplest arrays. The noncolinear arrays were already able to provide vectorial and anisotropy information. The focused arrays allowed a deeper investigation, because the current is focused at a greater depth than it would be possible by using simple electrodes. The superposed arrays allow the study either of lateral or vertical changes (or both) at a site. The null arrays, occurring in each group, obtain information exclusively about the subsurface inhomogeneity and -anisotropy.

The classification we propose may open the way to new geoelectric arrays, providing hopefully improving responses to the infinite variety of field problems we may face. In searching new geoelectric arrays, this paper helps to avoid rediscovering the discovered. Finally, this collection of arrays establishes the possibility of systematic intercomparisons of arrays, on basis of various theoretical or practical aspects.

\section{ACKNOWLEDGMENTS}

Support was received from several Hungarian National Scientific Research Fund projects (T049604, T037694, TS408048 and NI61013ILO). S. Szalai started this classification project during his postdoctoral fellowship at ULP Strasbourg (obtained from French Ministry of Education). Several Russian-origin arrays were obtained from V. Shevnin (now at Mexican Petroleum Institute, Mexico City). Discussions with A. Ádám, J. Verõ (GGRI, Sopron), E. Takács (Miskolc University), and some Russian literatures both from J. Kiss (ELGI, Budapest) are also acknowledged.

\section{REFERENCES}

Dahlin, T., and Zhou, B. [2004] A numerical comparison of 2D resistivity imaging with 10 electrode arrays. Geophysical Prospecting, 52, 379-398.

Szalai, S., Szarka, L., Prácser, E., Bosch, F., Müller, I., and Turberg, P. [2002] Geoelectric mapping of near-surface karstic fractures by using null arrays. Geophysics, 67, 17691778.

Szalai, S., and Szarka, L. [2007] Collection and classification of surface geoelectric arrays. Geophysical Prospecting, (under revision)

Tsokas, G.N., Tsourlos, P.I., and Szymanski, J.E. [1997] Square array resistivity anomalies and inhomogeneity ratio calculated by the finite element method. Geophysics, 62, 426435.

Van Nostrand, R.G., and Cook, K.L. [1966] Interpretation of resistivity data. Geological Survey professional paper 499. US Government Printing Office, Washington

Whiteley, R.J. [1973] Electrode arrays in resistivity and I.P. prospecting: a review. Bull. Aust. Soc. Explor. Geophys., 4, 1-29. 